\title{
Laparoscopic-assisted vaginal radical hysterectomy (LAVRH) in recipients of kidney transplant with cervical cancer stage Ib1
}

\author{
Giovanni Favero • Malgorzata Lanowska • \\ Achim Schneider • Simone Marnitz • \\ Christhardt Köhler
}

Received: 30 August 2009/Accepted: 17 November 2009/Published online: 12 December 2009

(C) Springer-Verlag 2009

\begin{abstract}
The incidence of cancer among patients submitted to kidney transplantation is increasing, since the graft survival rates have improved. Cervical cancer is approximately fivefold more common among these patients, correlated to immunosuppression exposure and antibody response to HPV infection. Treatment is challenging, regarding the graft in the pelvic field and standard options for stage Ib include radical hysterectomy or chemoradiation. We report two patients submitted to kidney transplantation with cervical cancer that were successfully treated by means of surgery. In conclusion, we intended to enlarge the current experience with radical hysterectomy in transplanted kidney patients with early stage cervical cancer. Although surgical difficulties existed, we could attest the feasibility and oncologic safety of the method.
\end{abstract}

Keywords Kidney transplantation · Cervical cancer . Radical hysterectomy

\section{Introduction}

Long-term complications of kidney transplantation have assumed an increasing importance, since the graft survival rates have improved. Recipients of a kidney transplant are

G. Favero · M. Lanowska • A. Schneider $(\bowtie) \cdot$ C. Köhler

Department of Gynecology, Charite Universitätsmedizin Berlin, Campus Mitte and Benjamin Franklin,

10117 Berlin, Germany

e-mail: achim.Schneider@charite.de

S. Marnitz

Department of Radiooncology, Charite Universitätsmedizin, Berlin, Germany at increased risk of malignancy when compared with general population [1]. The cancer risk increases with duration of survival after transplant, with a peak of around $30 \%$ after 15 years [2] It is difficult to precisely define the incidence of cervical cancer among these patients, but it is approximately fivefold more common [1].

The etiology is multifactorial, specially correlated to immunosuppression overall exposure [2]. It restricts the capacity of body to control the development of neoplasms, mainly the susceptibility to HPV infection [3].

Treatment of cervical cancer in the imunosupressed transplant patients is difficult especially regarding the graft in the pelvic field. Standard therapy options for stage $\mathrm{Ib}$ cervical cancer include radical hysterectomy or chemoradiation. Surgery can be complicated by prior ureteral operations, location of the transplant in the iliac fossa, and the renal vessels anastomose. On the other hand, clinical management may lead to a loss of renal function [3].

We intend to describe two patients submitted to kidney transplantation with cervical cancer who were successfully treated by means of surgery at our institution.

\section{Material and methods}

Case A A 47-year-old female received a cadaveric renal transplantation for renal hypoplastia at the age of 42 . The kidney was placed in the left iliac fossa, and the renal vessels were grafted to the external iliac vessels. Immunosuppression was based on Tacrolimus and Prednisolone, and renal function was normal.

Conization histology demonstrated a moderated differentiated squamous cell carcinoma (SCC), $10 \mathrm{~mm}$ (wide) and $4 \mathrm{~mm}$ (deep) - pT1b1. Preoperatively, the three ureters 
were catheterized through cystoscopy. Initially, a bilateral pelvic laparoscopic lymphadenectomy was performed, 20 lymph nodes were identified (12 right and eight left) and through frozen section analyzed. No nodal metastasis was detected, and consequently, a laparoscopic-assisted vaginal radical hysterectomy (LAVRH) could be done. The operative time and blood loss were $221 \mathrm{~min}$ and $120 \mathrm{ml}$, respectively. No surgical complication was observed.

The postoperative period was uneventful, and kidney function was stable. Complementary histology showed additional $8 \mathrm{~mm}$ tumor foci in uterine cervix and the resection margins were free. No adjuvant treatment was recommended. After a 1-year follow-up, the patient exhibits no signs of tumor recurrence.

Case B A 31-year-old woman received a cadaveric kidney transplantation for renal hypoplasia at the age of 14 . The kidney was placed in the left iliac fossa, and vessels were connected to the external iliac vessels. Immunosuppression was maintained with Mycophenolate mofetil and Predinisolone, and renal function was normal.

Conization histology revealed a 7-mm (wide) and 6-mm (deep) well-differentiated SCC (pT1b1). Stents were preoperatively placed in the three ureters through cystoscopy. A LAVRH was done after a pelvic lymphadenectomy. Eighteen negative lymph nodes were removed (11 right and seven left). The operation was uneventful, duration was $183 \mathrm{~min}$, and blood loss was $80 \mathrm{ml}$.

Renal function and postoperative kidney ultrasoundDoppler showed no abnormalities. Pathology of the surgical specimen revealed no residual tumor, and no adjuvant treatment was recommended.

The LAVRH consists of four parts: (a) catheterization of the three ureters; (b) lymphadenectomy and dissection of the vesicocervical-vesicovaginal septum by laparoscopy to evaluate nodal status or tumor involvement of adjacent organs (bladder, rectum) or abdominal cavity; (c) transvaginal preparation of a tumor-adapted vaginal cuff; and (d) laparoscopic radical hysterectomy, classified as Piver type II according to the oncologic radicality. After pneumoperitoneum was obtained, five laparoscopic trocars were placed: a $10 \mathrm{~mm}$ trocar was inserted at the level of the umbilicus, holding optics and camera; two $5 \mathrm{~mm}$ trocars were introduced in each lower quadrant of the abdomen; a 5-mm trocar was placed in the midline suprapubically; and a 10-mm trocar was placed in the left midclavicular line, 3$4 \mathrm{~cm}$ above the level of the umbilicus, for introducing an endoscopic bag to remove lymph nodes. The first conventional laparoscopic part consisted of inspection of the abdominal cavity and its organs and peritoneal washing with pelvic lymphadenectomy. For the vaginal part of radical hysterectomy, the patient was placed in the lithotomy position. In the transvaginal phase, a tumor- adapted vaginal cuff was created, and the vesicocervical and rectovaginal spaces were opened. In the last part, the uterine vessels and the medial part of the supraurethral bladder pillar were transected. The ureter was freed to allow its entry into the bladder without using a manipulator (Fig. 1). The entire extent of the remaining parts of the rectal pillar and the entire uterosacral-cardinal ligament complex were identified, coagulated, and cut under permanent visualization of the ureter. The suture of the vaginal cuff marked the endpoint of the resection. The specimen was removed via the vagina, and the vagina was closed with the previously placed single sutures.

\section{Conclusion}

Currently, there is an absence of data to support standard therapy for early stage cervical cancer in this group of patients. The cancer treatment impact on renal function, interaction between agents and immunosuppression, and oncologic safety should be taken into consideration to decide for the best therapy alternative.

Radical hysterectomy associated to lymphadenectomy may be technically complicated, and the graft in the pelvic position poses a number of operative difficulties. According to some authors, this can limit the possibility to perform an adequate lymphadenectomy and parametrial dissection, as well as putting into risk the ureter, and transplant vascular supply [3]. Conversely, chemoradiation may lead to renal function impairment, and its use in these patients is limited to two case reports $[3,4]$.

In the presented cases, the number of removed nodes, the length of parametrial dissection, and vaginal cuff can be considered satisfactory, which can attest the oncologic quality of the surgery. Associated to the fact that operation

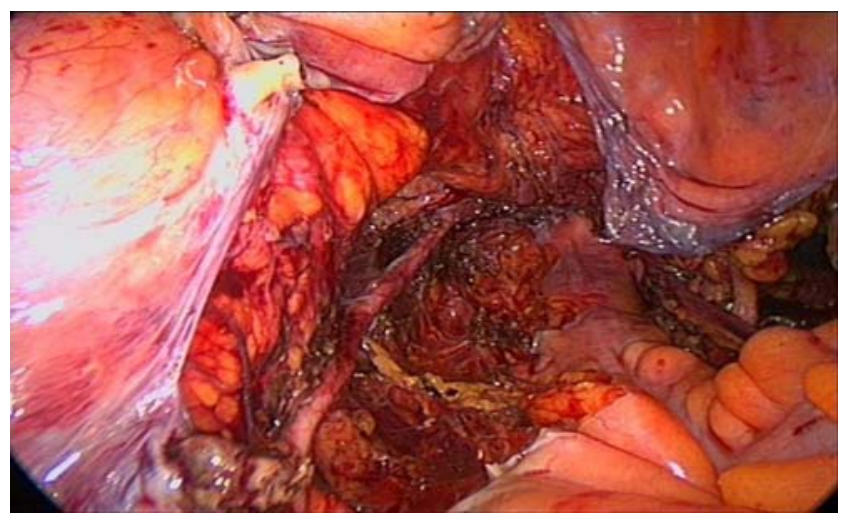

Fig. 1 Laparoscopic view of pelvic region after the uterus and lymph nodes removal. On the left side, transplanted kidney and the route of the ureter 
time and blood loss were also optimal. Moreover, we did not observe any type of intra or postoperative complication and renal function after the procedure in both cases were stable.

It is important to underline the preoperative ureteral catheterization in order to increase surgical safety and to reduce resulting injuries. Its use facilitates the ureter intraoperative identification and also repair, when a complication occurs.

In conclusion, we intended to enlarge the current literature experience with radical hysterectomy in transplanted kidney patients. To date, there is only one report of a radical hysterectomy performed in recipients of kidney transplantation as treatment for early stage cervical cancer [5]. Although surgical difficulties, we could attest the feasibility and oncologic safety of the procedure, and we believe that this therapy option should ever be considered.
Conflict of interest statement The authors declare no conflict of interest.

\section{References}

1. Kasiske BL, Snyder JJ, Gilbertson DT, Wang C (2004) Cancer after kidney transplantation in the United States. Am J Transplant 4 (6):905-913

2. Kapoor A (2008) Malignancy in kidney transplant recipients. Drugs 68(Suppl 1):11-9 Review

3. Ripley D, Levenback C, Eifel P, Lewis RM (1995) Adenocarcinoma of the cervix in a renal transplant patient. Gynecol Oncol 59 (1):151-5

4. Tao X, Kavanagh JJ (2008) Chemotherapy for gynecological malignancies in organ transplantation patients: report of two cases. Int J Gynecol Cancer 18(6):1376-80 Epub 2008 May 6

5. Raff GJ, Kasper KM, Hollinger EF Jr, Goggins WC (2008) Laparoscopic hysterectomy in patients with prior renal transplantation. J Minim Invasive Gynecol 15(2):223-6 